\title{
Multi-loop PID Control Method of Brushless DC Motors via Convex Combination Method
}

\author{
Chang-Hyun Kim ${ }^{\dagger}$
}

\begin{abstract}
This paper proposes the explicit tuning rule of multi-loop PID controller for brushless direct current motors to predict the system behaviors in time and frequency domains, using properties of the convex combination method. The convex set of the proposed controllers formulates the envelope to satisfy the performances in time and frequency domains. The final control parameters are determined by solving the convex optimization problem subject to the constraints which are represented as convex set of time domain performances. The effectiveness of the proposed control method is shown in the numerical simulation, in which controller tuning algorithm and dynamics of brushless DC motor are well taken into account.
\end{abstract}

Keywords: BLDC, PID, PI-PD, Convex set, Convex combination method, LMI

\section{Introduction}

Brushless Direct Current (BLDC) motors have been widely applied in various industries due to their advantages such as higher efficiency, reliability, starting torque, reduced electrical noises, and so on. [1]. While serieswound brushed DC motors are applied for traction motors and starters of trains and railway vehicles, typical applications of BLDC motors are urban rail facilities such as railway carriage fans, screen doors, and so on [2]. Recently, various BLDC motors are studied for urban rail traction and drive train system of electric vehicle with in wheel motors [3, 4].

Although various modern control theories have been developed for the speed tracking control of BLDC motors, conventional proportional integrate differential (PID) controller is most commonly used in industry owing to their merits of simple structure, high efficiency and easy implementation [5]. However, the optimal tuning of PID controllers is still difficult to obtain proper step responses [6]. After initial tuning such as Ziegler-Nichols, CohenCoon methods, genetic algorithm, Linear Quadratic (LQ)PID, and particle swam optimization (PSO), the trial and error way is generally applied in practice [7].

In this paper, an enhanced tuning method of multi-loop PID controller with an internal feedback controller is proposed for speed control of BLDC motor, called as twodegree of freedom controller [8]. The proposed PID controller has an additional inner-loop controller to conventional PID controller in order to formulate the convex combination set between transfer functions of the initial controllers.

The time- and frequency-responses in the convex set

$\dagger$ Corresponding Author: Dept. of Electricity, VISION College of

Jeonju, Korea. (chkim@jvision.ac.kr)

Received: January 25, 2016; Accepted: March 7, 2016 are explicitly followed on the convex properties such that the engineer can have the intuition to predict the controlled results [9]. After making the initial boundary of the set, the controller is designed in order to satisfy the specifications in time and frequency domains. The boundary is taken into account as an envelope curve to limit the response of the controlled system in order to meet the specifications in time- and frequency domains. Differently from the structure of a conventional PID controller, the proposed advanced controller can be modified as the various formulations according to inner-loop compensator [10].

The structure of the proposed controller is organized as the convex combination of the conventional PID controllers satisfying the time- and frequency-domain performances. The combination parameters are obtained by solving the linear matrix inequality (LMI) optimization problem subject to the convex constraints. In this paper, the conventional PID controller with an internal feedback compensator is chosen in order to develop the convex combination. The effectiveness of the proposed control method is shown in the simulation to realize the BLDC motor and actuator.

The main contributions of the proposed method are summarized as follows:

(i) The proposed method provides the intuition to control engineers to expect the controlled motor speed by time and frequency domain properties of convex combination method.

(ii) The control parameters are explicitly determined by the proposed LMI optimization problem.

(iii) The proposed multi-loop PID controller has degreeof-freedom to consider various design performances in time-and frequency-domains.

\section{BLDC Motor Model}

In this chapter, the general dynamics of BLDC motors is 
briefly introduced, which is not totally different from the conventional DC motors. The mathematical model of BLDC motor drive is simplified by a few assumptions that (1) symmetrical three-phase winding, (2) no magnetic saturation, (3) no hysteresis and eddy current losses, (4) uniform air-gap, (5) mutual inductance is ignored, and (6) armature reaction is ignored [10]. Mathematical voltage equation of armature winding is described as

$$
u_{\text {app }}(t)=R i(t)+L \frac{d i(t)}{d t}+u_{e m f}(t)
$$

where $u_{a p p}(t)$ (terminal voltage $\left.[V]\right), i(t)$ (stator current $[A]), u_{e m f}(t)$ (counter electromotive force), $L$ (armature self-inductance), $R$ (armature resistance), The mechanical equation is represented by the sum of all torques as follows:

$$
J \frac{d \omega(t)}{d t}=\sum \tau_{i}
$$

All torques $\tau_{i}$ are

$$
J \frac{d \omega(t)}{d t}=K_{m} \cdot i(t)-T_{L}-K_{f} \omega(t)
$$

where $J\left(\right.$ rotor inertia $\left.\left[\mathrm{kg} \cdot \mathrm{m}^{2}\right]\right), \omega(t)$ (rotor speed $\left.[\mathrm{rad} / \mathrm{sec}]\right)$, $K_{m}$ (torque constant $[\mathrm{kg} \cdot \mathrm{m} / \mathrm{A}]$ ), $T_{L}$ (load torque $[\mathrm{Nm}]$ ), $K_{f}$ (friction constant $[\mathrm{kg} \cdot \mathrm{m} \cdot \mathrm{s}]$ ), $u_{\text {emf }}(t)$ is proportional to the angular velocity with the back EMF constant $K_{b}[\mathrm{~kg} \cdot \mathrm{m} \cdot \mathrm{s} / \mathrm{rad}]$ as the follows:

$$
u_{e m f}(t)=K_{b} \cdot \omega(t)
$$

The state-space equation of BLDC motor can be rewritten as a second order ordinary differential equation with respect to the state and input variables such that

$$
\begin{aligned}
& \frac{d}{d t}\left[\begin{array}{c}
i(t) \\
\omega(t)
\end{array}\right]=\left[\begin{array}{cc}
-\frac{R}{L} & -\frac{K_{b}}{L} \\
\frac{K_{m}}{J} & -\frac{K_{f}}{J}
\end{array}\right]\left[\begin{array}{c}
i(t) \\
\omega(t)
\end{array}\right]+\left[\begin{array}{c}
\frac{1}{L} \\
0
\end{array}\right] u_{a p p}(t) \\
& y(t)=\left[\begin{array}{ll}
0 & 1
\end{array}\right]\left[\begin{array}{c}
i(t) \\
\omega(t)
\end{array}\right] .
\end{aligned}
$$

where the states are the current $i(t)$ and the angular velocity $\omega(t)$, input variable is the applied voltage $u_{a p p}(t)$, and the measured output $y(t)$ is the angular velocity.

The transfer function from the input to the output is derived as follows:

$$
G(s)=\frac{W(s)}{U(s)}=\frac{K_{m}}{J L s^{2}+\left(R J+K_{f} L\right) s+R K_{f}+K_{b} K_{m}}
$$

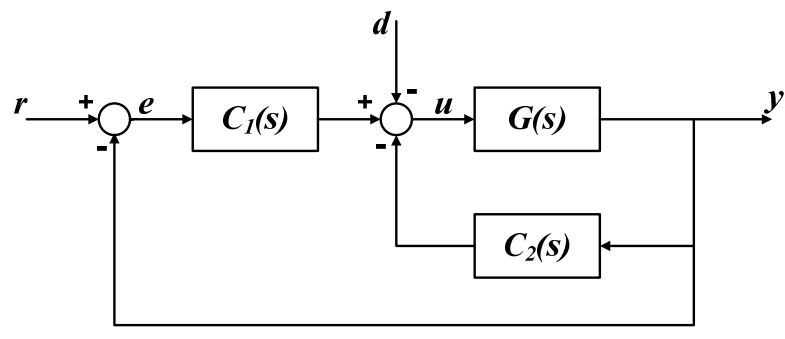

Fig. 1. Structure of 2 DOF Control System

\section{Multi-loop PID Control Scheme}

In this chapter, the structure of multi-loop feedback control systems with an inner feedback loop is introduced, called as two degree of freedom (DOF) controller, shown in Fig. 1.

Fig. 1 illustrates the general input output signals and block diagram, where $G(s), C_{1}(s), C_{2}(s), r, u, y, d$, and $e$ indicate the nominal plant model, the controllers, the reference input, the control command, the plant output, the disturbance signal applied to the system, and the error defined as $e=r-y$, respectively [11].

The complementary sensitivity function of the inputoutput relation is expressed as Eq. (7)

$$
H_{r y}(s)=\frac{C_{1}(s) G(s)}{1+\left(C_{1}(s)+C_{2}(s)\right) G(s)} .
$$

If $C_{2}(s)$ is removed as zero and $C_{1}(s)$ is PID controller, the closed loop system is exactly same to the conventional PID control system. According to the considered controller for $C_{2}(s)$, various controlled systems can be formulated

In this paper, PID and PI-PD controllers are dealt with to formulate the upper and lower bounds of the convex set. The traditional PID controller is denoted as

$$
\begin{gathered}
C_{1}(s)=K_{p}+K_{i} \frac{1}{s}+K_{d} s, \\
C_{2}(s)=0 .
\end{gathered}
$$

The transfer functions of $C_{1}(s)$ and $C_{2}(s)$ indicate PI controller with PD inner controller as follows :

$$
\begin{aligned}
& C_{1}(s)=K_{p 1}+K_{i 1} \frac{1}{s}, \\
& C_{2}(s)=K_{p 2}+K_{d 2} s .
\end{aligned}
$$

\section{CS-based PID-PD Controller}

For reading comprehension, several mathematical definitions should be introduced in this chapter. H, D, and $H$ denote the set of all closed-loop transfer matrices, 
Table 1. Mathematical definition for convex set

\begin{tabular}{c|c}
\hline Design specifications & Sets of transfer matrices \\
\hline$H$ satisfies D & $H \in \mathrm{H}$ \\
$\mathrm{D}_{1}$ is stronger than $\mathrm{D}_{2}$ & $\mathrm{H}_{1} \subseteq \mathrm{H}_{2}$ \\
$\mathrm{D}_{1}$ is weaker than $\mathrm{D}_{2}$ & $\mathrm{H}_{1} \supseteq \mathrm{H}_{2}$ \\
$\mathrm{D}_{1} \wedge \mathrm{D}_{2}$ & $\mathrm{H}_{1} \cap \mathrm{H}_{2}$ \\
$\mathrm{D}_{1}$ is infeasible & $\mathrm{H}_{1}=0$ \\
$\mathrm{D}_{1}$ is feasible & $\mathrm{H}_{1} \neq 0$ \\
\hline
\end{tabular}

design specifications, and one element of transfer matrices set $\mathrm{H}$, respectively. $[9,10]$

$\mathrm{H}$ indicates the set of all conceivable candidate transfer matrices for the given plant to satisfy each design specification $\mathrm{D}_{i}$ associated to the set $\mathrm{H}_{i}$ of all transfer matrices $[9,10]$ :

$$
\mathrm{H}_{i}=\left\{H \in \mathrm{H} \mid \mathrm{H} \text { satisfies } \mathrm{D}_{i}\right\} .
$$

The definition 1 and 2 are reintroduced to present that a set of transfer matrices satisfies the specifications if two distinct transfer matrices formulate the convex set according to convex combination method.

Definition 1: $\mathrm{H}_{1} \subseteq \mathrm{H}$ is affine if for any $H, \tilde{H} \in \mathrm{H}_{1}$, and any $\lambda \in R, \lambda H+(1-\lambda) \tilde{H} \in \mathrm{H}_{1}$, where if $\lambda$ is restricted within $[0,1]$, the affine combination $\lambda H+(1-\lambda) \tilde{H}$ is in the convex set $[9,10]$.

Definition 2: A functional $\varphi$ on $\mathrm{H}$ is convex if for any $H, \tilde{H} \in \mathrm{H}$ and any $\lambda \in[0,1], \varphi(\lambda H+(1-\lambda) \tilde{H}) \leq$ $\lambda \varphi(H)+(1-\lambda) \varphi(\tilde{H})[9,10]$.

The convex set properties can be applied in order to design the general feedback controller. If the general unit feedback controller, $H_{\text {pro }}$ is designed to formulate the convex set between $H_{\mathrm{ry}}$ and $\tilde{H}_{\mathrm{ry}}, H_{\text {pro }}$ is controlled by the controller $C_{\text {pro }}$ in Eq. (15)

where

$$
\begin{gathered}
H_{p r o}(s)=\frac{C_{p r o}(s) G(s)}{1+C_{p r o}(s) G(s)} \\
H_{r y}(s)=\frac{C_{1}(s) G(s)}{1+C_{1}(s) G(s)} \\
\tilde{H}_{r y}(s)=\frac{\tilde{C}_{1}(s) G(s)}{1+\tilde{C}_{1}(s) G(s)} \\
H_{p r o}(s)=\lambda H_{r y}(s)+(1-\lambda) \\
\tilde{H}_{r y}(s)=\frac{C_{p r o}(s) G(s)}{1+C_{p r o}(s) G(s)} .
\end{gathered}
$$

The controller $C_{\text {pro }}$ is obtained as Eq. (16) from component to form the convex set.

$$
C_{p r o}(s)=\frac{-\left(\lambda H_{r y}(s)+(1-\lambda) \tilde{H}_{r y}(s)\right)}{\left(\lambda H_{r y}(s)+(1-\lambda) \tilde{H}_{r y}(s)-1\right) G(s)} .
$$

Thus, the designed controller is represented as Eq. (17)

$$
C_{p r o}(s)=\frac{\lambda C_{1}-\lambda \tilde{C}_{1}+\tilde{C}_{1}+C_{1} \tilde{C}_{1} G}{1+C_{1} G-\lambda C_{1} G+\lambda \tilde{C}_{1} G}
$$

such that the order of the desired controller should be dependent on the plant. This dependency problem is very complicated to design the controller in practice.

In this paper, the proposed controller is independent on the order of the plant because the controlled system, represented as Eq. (18) has the common denominator with respect to the convex set.

$$
\begin{aligned}
T_{P I D-P D} & =\frac{C_{P I D}(s) G(s)}{1+\left(C_{P I D}(s)+C_{P D}(s)\right) G(s)} \\
& =\lambda_{1} \frac{C_{p i}(s) G(s)}{1+\left(C_{p i}(s)+C_{p d}(s)\right) G(s)}+\lambda_{2} \frac{C_{p i d}(s) G(s)}{1+C_{p i d}(s) G(s)}
\end{aligned}
$$

In the design scheme, the proposed PID-PD controller is determined to form convex combination from PI-PD, represented as Eq. (19) and PID-PD, represented as Eqs. (20) and (21), respectively

$$
\begin{gathered}
C_{p i}(s)=\frac{\beta}{1+\beta} K_{p}^{*}+\frac{T_{i}^{*}}{s}, \quad C_{p d}(s)=\frac{1}{1+\beta} K_{p}^{*}+T_{d}^{*} s \\
C_{P I D}(s)=\frac{\lambda_{1} \beta+\lambda_{2}}{1+\beta} K_{p}^{*}+\lambda_{1} \frac{T_{i}^{*}}{s}+\lambda_{2} T_{d}^{*} s, \\
C_{P D}(s)=\frac{\lambda_{2} \beta+\lambda_{1} K_{p}^{*}}{1+\beta}+\lambda_{1} T_{d}^{*} s .
\end{gathered}
$$

At $\lambda_{1}=\lambda, \lambda_{2}=1-\lambda$, the step responses of the proposed control system show the convex properties in Fig. 2, such that the boundary of convex set formulates the envelop curve of the desired response [11]. The PI$\mathrm{PD}$ controller is determined by control factor $\beta$ to set the lower boundary of the desired controlled response.

To determine the control parameters, $\lambda_{1}$ and $\lambda_{2}$, of the proposed controller, three functionals of $H$ are dealt with in the time-domain specifications such as the overshoot, rise-time, and settling time in this paper.

These functionals on $H$ are convex, so the functional inequality specifications,

$$
\begin{gathered}
\mathrm{D}_{\mathrm{os}}^{\left(\mathrm{a}_{1}\right)}: \varphi_{O S}(H) \leq \alpha, \\
\mathrm{D}_{\text {rise }}^{\left(\mathrm{a}_{2}\right)}: \varphi_{\text {rise }}(H) \leq T_{\max } \\
\mathrm{D}_{\text {settle }}^{\left(\mathrm{a}_{3}\right)}: \varphi_{\text {settle }}(H) \leq T_{\text {max_set }}
\end{gathered}
$$

are convex $[9,10]$.

The convex functional inequalities of the given design specifications can be represented as linear matrix inequalities. The control gains are obtained by solving the convex optimization problem, in which the objective 


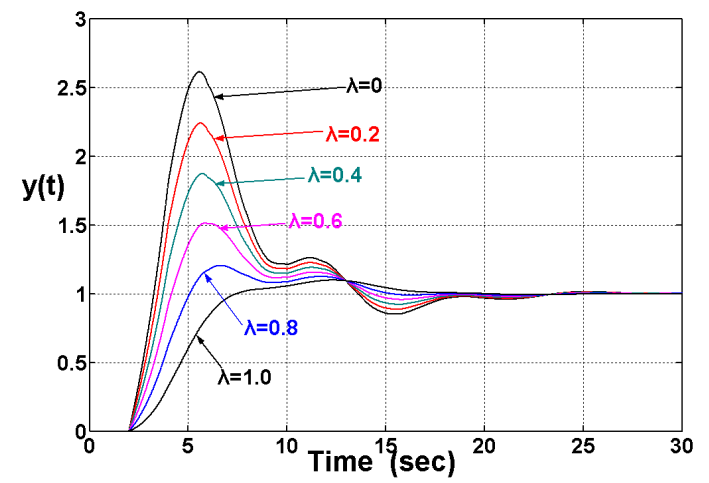

Fig. 2. Aspect of step responses obtained from convex set of the controlled systems

functional can be formed as a weighted-sum objective

$$
\varphi_{o b j}(H)=b_{1} \varphi_{1}(H)+\cdots+b_{L} \varphi_{L}(H)
$$

where $b_{i}$ are weighting factors, which the designer selects according to the importance of the performances.

Thus, the general convex optimization problem to design the proposed controller is represented as

$$
\begin{aligned}
& \text { minimize } \varphi_{o b j}(\lambda)=b_{1} \varphi_{1}(\lambda)+\cdots+b_{L} \varphi_{L}(\lambda) \\
& \text { subject to } \varphi_{1}(H) \leq a_{1}, \ldots, \varphi_{L}(H) \leq a_{L} \\
& \text { bounded to } o \leq \lambda_{i} \leq 1(i=1,2, \ldots), \sum \lambda_{i}=1
\end{aligned}
$$

where the constraints are determined in order to formulate the envelop curve of the design performances.

In this paper, the convex optimization is derived as Eq. (27) subject to the constraints which describe the design specifications as LMI with respect to overshoot, rise-time, and settling time.

$$
\begin{array}{ll}
\operatorname{minimize} & \varphi_{o b j}(\lambda)=b_{1} \alpha_{O S}+b_{2} \beta_{r t}+b_{3} \gamma_{s t} \\
\text { subject to } & {\left[\begin{array}{cc}
\alpha_{\mathrm{OS} 1} & \alpha_{\mathrm{OS} 2} \\
\beta_{\mathrm{r} \mathrm{1} 1} & \beta_{\mathrm{r} \mathrm{2} 2} \\
\gamma_{s t 1} & \gamma_{s t 2}
\end{array}\right]\left[\begin{array}{l}
\lambda_{1} \\
\lambda_{2}
\end{array}\right] \leq\left[\begin{array}{l}
\alpha_{\text {desire }} \\
\beta_{\text {desire }} \\
\gamma_{\text {desire }}
\end{array}\right]}
\end{array}
$$

bounded to $o \leq \lambda_{i} \leq 1(i=1,2), \quad \sum \lambda_{i}=1$

In order to design the controller, first the region of the feasible controllers as the envelop curve is bounded to satisfy the constraints of the system specifications. Secondly, the optimization problem is applied to find the optimum value of the weighted-sum objective functional as Eq. (27), subject to the convex set to satisfy the functional inequality specifications. Finally, the parameters of PIDPD controller are obtained by solving the optimization problem. Fig. 3 summarizes the design procedure of the proposed controller.

The design procedure of the proposed method is summarized as

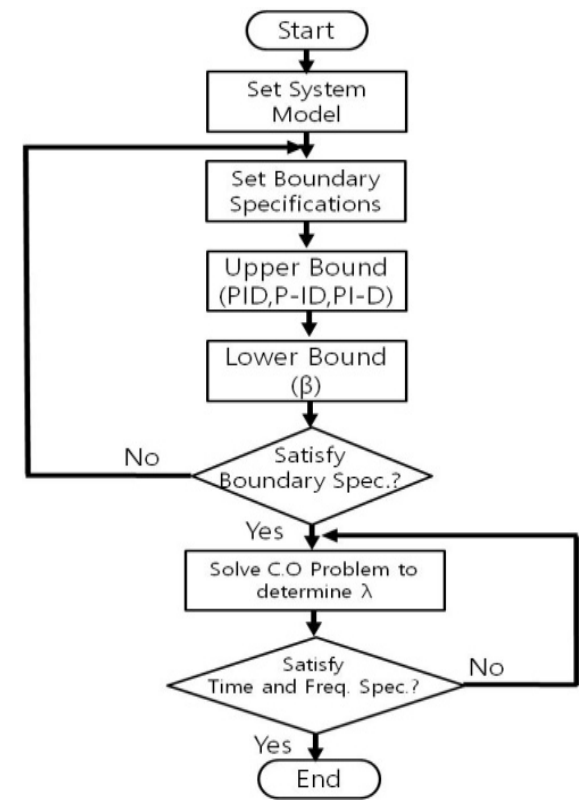

Fig. 3. Control design flow-chart

\section{Summary of Design Procedure}

Step 1: Set model parameters of a given plant

Step 2: Specify the boundary satisfying the required design specifications such as overshoot, rise-time, settling time, and so on.

Step 3: Choose the objective functional to weight the considered performances according to the system requirement.

Step 4: Solve the convex optimization problem subject to constraints and calculate the control gains.

Step 5: Finish the process to determine the adequate controller if all of required design specifications are satisfied, if not reset the constraints, beginning with Step 2 .

\section{Simulation}

In controller tuning algorithm, mechanical and electrical dynamics of BLDC motor are taken into account by simulation test.

The specifications of the BLCD motor are shown in Table 2. The transfer function of the BLDC motor is obtained as Eq. (22). [10]

Table 2. System parameters of the BLDC motor

\begin{tabular}{c|c}
\hline Parameters & Values and units \\
\hline$R$ & $21.2 \Omega$ \\
\hline$K_{b}$ & $0.1433 \mathrm{~V} \mathrm{~s} / \mathrm{rad}$ \\
\hline$K_{f}$ & $1 \times 10^{-4} \mathrm{~kg}-\mathrm{m} \mathrm{s} / \mathrm{rad}$ \\
\hline$L$ & $0.052 \mathrm{H}$ \\
\hline$K_{m}$ & $0.1433 \mathrm{~kg}-\mathrm{m} / \mathrm{A}$ \\
\hline$J$ & $1 \times 10^{-5} \mathrm{~kg}-\mathrm{m} \mathrm{s} / \mathrm{rad}$ \\
\hline
\end{tabular}




$$
G(s)=\frac{275577.36}{s^{2}+417.7 s+43567.5}
$$

The optimization problem for this example is determined as

$$
\begin{aligned}
& \operatorname{minimize} \quad \varphi_{o b j}(\lambda)=0.2 \alpha_{O S}+3.3 \beta_{r t}+1.4 \gamma_{s t} \\
& \text { subject to }\left[\begin{array}{cc}
\alpha_{\mathrm{OS} 1} & \alpha_{\mathrm{OS} 2} \\
\beta_{\mathrm{rt} 1} & \beta_{\mathrm{rt} 2} \\
\gamma_{s t 1} & \gamma_{s t 2}
\end{array}\right]\left[\begin{array}{l}
\lambda_{1} \\
\lambda_{2}
\end{array}\right] \leq\left[\begin{array}{l}
5 \% \\
0.3 m s \\
0.7 m s
\end{array}\right] \\
& \text { bounded to } o \leq \lambda_{i} \leq 1(i=1,2), \sum \lambda_{i}=1
\end{aligned}
$$

where the weighting factors of the cost function are selected in order to regulate each performances without weights such that the specifications with respect to $\alpha_{O S}$, $\beta_{r t}, \gamma_{s t}$ are considered with the exactly same importance.

The various tuning methods of PID such as LQ-PID, POS, and genetic algorithms are chosen to compare the time-domain response of the proposed control system with the conventional PID methods $[12,13]$. As shown Fig. 4 and Table 3, the proposed method only satisfies the requirement of the time-domain specification.

The proposed method results that the design specifications such as the overshoot, rise-time, and settling time not only maintain into the boundary, but it achieves the better speed performances comparing with the other methods in this case.

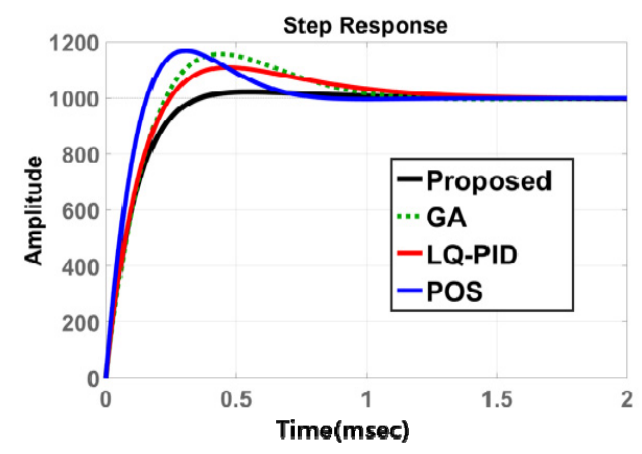

Fig. 4. Comparison of step responses

Table 3. Comparison of system performances

\begin{tabular}{c|c|c|c}
\hline Methods & $\begin{array}{c}\text { Rise time } \\
(\mathrm{msec})\end{array}$ & $\begin{array}{c}\text { Settling time } \\
(\mathrm{msec})\end{array}$ & $\begin{array}{c}\text { Overshoot } \\
(\%)\end{array}$ \\
\hline Proposed & 0.223 & 0.639 & 2.16 \\
\hline GA & 0.185 & 0.982 & 15.6 \\
\hline LQ-PID & 0.177 & 1.14 & 10.8 \\
\hline PSO & 0.128 & 0.69 & 16.8 \\
\hline
\end{tabular}

\section{Conclusion}

In this paper, a novel tuning formula for the BLDC motors was proposed in order to obtain a good command following properties via convex combination method formulating the convex set between PID and PI-PD controllers. By solving the derived convex optimization problem subject to LMI constraints, the PID-PD control gains are determined, minimizing the time-domain performances which have convex set properties. The advantages of the proposed method are that all tuning parameters are analytically obtained according to the optimization solution, the stability of the controlled system is guaranteed inherently from the convex set properties, and various time-domain performances can be dealt with by adding the controllers to provide the convex set.

For future works, it needs to research for the generality of the proposed method in the areas such as the multivariable system, nonlinear applications, and so on.

\section{References}

[1] D. M. Dawson, J. Hu and T. C. Burg, Nonlinear control of electric machinery, Marcel Dekker, Inc., 1998.

[2] S. W. Fardo and R. P. Dale, Electrical power systems technology. The Fairmont Press, Inc., 2009.

[3] A. Tashakori and E. Mehran,. "Fault diagnosis of inwheel bldc motor drive for electric vehicle application." Intelligent Vehicles Symposium (IV), 2013 IEEE, 2013.

[4] W. Min and J. Wei, "Control study of rare earth permanent brushless DC motor in city rail." Computer Science and Automation Engineering (CSAE), 2011.

[5] A. Mashakbeh and A. S. Othman, "Proportional Integral and Derivative Control of Brushless DC Motor." European Journal of Scientific Research, vol. 35, no. 2, pp. 198-203, 2009.

[6] C. L. Lin and H. Y. Jan, "Multi-objective PID control for linear brushless DC motor: an evolutionary approach," IEE Proceedings-Electric Power Applications, vol. 149, no. 6, pp.397-406, 2002.

[7] Shahrokhi, Mohammad, and Alireza Zomorrodi. "Comparison of PID controller tuning methods." Department of Chemical \& Petroleum Engineering, Sharif University of Technology, 2013.

[8] C. H. Kim, D. G. Lim and B. S. Suh, "Optimum tuning of PID-PD controller considering robust stability and sensor noise insensitivity," Journal of The Institute of Electronics Engineers of Korea, vol. 44, no. 1, pp. 19-24, 2007.

[9] S. Boyd and C. Barratt, Linear Controller : Limits of Performance. Prentice Hall, Englewood Cliffs, NJ., 1991.

[10] C. H. Kim, J. H. Yang, D. G. Lim, and B. S. Suh, "An Enhanced PID Controller for Speed Control of Brushless DC Motors Based on Convex Set Optimization." IFAC Proceedings Volumes, vol. 43, no. 
22, pp. 75-80, 2010.

[11] C. H. Kim, "Optimum Tuning Method for PID-PD Controllers", MS Thesis, Hanyang Universiy, 2006.

[12] C. L. Cham, "Brushless DC Motor Electromagnetic Torque Estimation with Single-Phase Current Sensing", Journal of Electrical Engineering \& Technology, vol. 9, no. 3, pp. 866-872, 2014.

[13] M. Nasri and H. Nezamabadi-Pour, "A PSO-based optimum design of PID controller for a linear brushless DC motor." World Academy of Science, Engineering and Technology, vol. 26, no. 40, pp. 211-215, 2007.

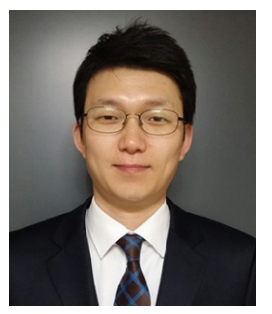

Chang-Hyun Kim He received his M.S and $\mathrm{Ph} . \mathrm{D}$ in Electrical Engineering from Hanyang University, Korea, in 2006, and 2015, respectively. He was a senior researcher at Korea Marine Equipment Research Institute from 2015 to 2016. He is currently an assistant professor at VISION College of Jeonju, Korea. His current research interests include the robust control, MPC, optimal digital control, and its application to electric power grid, network congestion control, magnetic levitation systems, and so on. 